\title{
New Stable Manganese(III) Chelates of Some Pyridine Carboxylic Acids
}

\author{
Saktiprosad Ghosh*, Pranab K. Ray, Tapas K. Bandyopadhyay, and Amal K. Deb \\ Department of Inorganic Chemistry, Indian Association for the Cultivation of Science, \\ Jadavpur, Calcutta 700032, India
}

Z. Naturforsch. 36 b, 1270-1272 (1981); received June 10, 1981

Manganese(III) Chelates

The syntheses of three new and unusually stable Mn(III) complexes of pyridine-2carboxylic acid, pyridine-2,6-dicarboxylic acid and pyridine-2,4,6-tricarboxylic acid and their characterisation by various standard physicochemical methods is reported.

\section{Introduction}

$\mathrm{Mn}$ (III) complexes stable in aqueous solution as well as in the solid state are rare [1-6]. The complexes that we have synthesised are tris-(pyridine-2. carboxylato)manganese(III), (A), bis-(pyridine-2,6dicarboxylato)manganese(III), (B) and bis-(pyridine-2,4,6-tricarboxylato)manganese(III), (C). Electronic and IR spectral characteristics along with elemental analyses and room temperature magnetic susceptibility data have been utilised to arrive at the possible ligand environment and the oxidation state of manganese. The +3 state of manganese has been established by oxidimetric methods using both acidified potassium iodide and standard Mohr salt solutions. Room temperature magnetic moments of all these chelates lie within the range of 3.90 to 4.00 B.M. and are characteristic of $\mathrm{Mn}$ (III) in an octahedral donor environment. Electronic absorption spectra of these chelates exhibit a broad band around $15,990-19,850 \mathrm{~cm}^{-1}$ indicative of the presence of $\mathrm{Mn}$ (III) in an octahedral ligand field. The IR spectrum of the first chelate shows evidences of the presence of only coordinated carboxylate moieties and of lattice water, while in the spectra of the other chelates, characteristic bands of both coordinated and uncoordinated carboxyl groups are present along with those of lattice water.

\section{Experimental}

\section{Reagents and chemicals}

All chemicals used were of A. R., B. D. H. grade or of equivalent quality. $\mathrm{Mn}$ (III) acetate was prepared by the standard method.

* Reprint requests to Dr. Saktiprosad Ghosh. $0340-5087 / 81 / 1000-1270 / \$ 01.00 / 0$

\section{Preparation of the complexes}

The complexes were prepared by the following two different procedures:

(i) Freshly precipitated manganese dioxide was warmed with an aqueous solution of excess pyridine carboxylic acids with vigorous stirring and filtered through a sintered glass funnel. The scarlet-red solution deposited red/reddish brown/brown crystals on cooling.

(ii) Finely powdered $\mathrm{Mn}$ (III) acetate was added to a warm concentrated aqueous solution of pyridine-carboxylic acids (excess), stirred well and filtered. On cooling the solution deposited red/reddish brown/brown crystals.

The crystals obtained by the above procedures were filtered, washed with water and dried over fused $\mathrm{CaCl}_{2}$.

The complex, tris(pyridine-2-carboxylato)manganese(III), (A), $\mathrm{Mn}\left(\mathrm{C}_{13} \mathrm{H}_{12} \mathrm{~N}_{3} \mathrm{O}_{6}\right) \cdot \mathrm{H}_{2} \mathrm{O}$, was also prepared by the following method. A solution of potassium permanganate was heated with a large excess of pyridine-2-carboxylic acid and filtered as above. The filtrate deposited deep-red shining crystals on cooling.

The other two complexes (B), $\mathrm{Mn}\left(\mathrm{C}_{14} \mathrm{H}_{7} \mathrm{~N}_{2} \mathrm{O}_{8}\right)$. $2 \mathrm{H}_{2} \mathrm{O}$ and (C), $\mathrm{Mn}\left(\mathrm{C}_{16} \mathrm{H}_{7} \mathrm{~N}_{2} \mathrm{O}_{12}\right) \cdot 2 \mathrm{H}_{2} \mathrm{O}$ were also prepared from the complex (A). The complex (A) was added to a hot aqueous solution of a slight excess of the respective pyridine-carboxylic acids, the red solution obtained was filtered hot and gradually cooled to room temperature. The compounds separated as reddish brown/brown granular solids.

\section{Analyses and physicochemical measurements}

Mn was analysed by the usual procedure whereas, nitrogen was estimated by semi-micro Dumas' method. Analyses of $\mathrm{C}, \mathrm{H}$ were performed by the Microanalytical Laboratory of our institute. Analytical data together with the colour of the complexes were tabulated in Table I. Magnetic susceptibility measurements were carried out on a Gouy balance at $298 \mathrm{~K}$ taking mercuric tetrathiocyanato 
Table I. Analytical results, colour, magnetic moment, spectral (visible) data for the complexes.

\begin{tabular}{|c|c|c|c|c|c|c|c|c|}
\hline \multirow[t]{2}{*}{ Complex } & \multirow[t]{2}{*}{ Colour } & \multicolumn{4}{|c|}{ Analysis* [\%] } & \multirow{2}{*}{$\begin{array}{l}\mu_{\text {efp }} \text { B.M. } \\
\text { at } 298 \mathrm{~K}\end{array}$} & \multirow{2}{*}{$\begin{array}{l}\lambda_{\max } \\
{\left[\mathrm{cm}^{-1}\right]}\end{array}$} & \multirow{2}{*}{$\begin{array}{l}\varepsilon \\
{\left[\mathrm{cm}^{-1} \mathrm{~mole}^{-1}\right]}\end{array}$} \\
\hline & & $\mathrm{C}$ & $\mathrm{H}$ & $\mathbf{N}$ & Mn & & & \\
\hline (A) & $\begin{array}{l}\text { Deep } \\
\text { red }\end{array}$ & $\begin{array}{c}41.09 \\
(41.16)\end{array}$ & $\begin{array}{c}3.74 \\
(3.69)\end{array}$ & $\begin{array}{c}9.49 \\
(9.54)\end{array}$ & $\begin{array}{c}12.44 \\
(12.51)\end{array}$ & 3.90 & $15,990-19,850$ & 40 \\
\hline (B) & $\begin{array}{l}\text { Reddish } \\
\text { brown }\end{array}$ & $\begin{array}{c}39.74 \\
(39.81)\end{array}$ & $\begin{array}{c}2.69 \\
(2.60)\end{array}$ & $\begin{array}{c}6.58 \\
(6.63)\end{array}$ & $\begin{array}{c}12.94 \\
(13.03)\end{array}$ & 4.0 & $16,000-19,600$ & 52 \\
\hline (C) & Brown & $\begin{array}{c}37.60 \\
(37.65)\end{array}$ & $\begin{array}{l}2.21 \\
(2.15)\end{array}$ & $\begin{array}{c}5.44 \\
(5.49)\end{array}$ & $\begin{array}{c}10.72 \\
(10.78)\end{array}$ & 3.97 & $16,600-18,800$ & 60 \\
\hline
\end{tabular}

* Theoretical values in parantheses.

cobalt(II), $\mathrm{Hg}\left[\mathrm{Co}(\mathrm{CNS})_{4}\right]$ as the callibrant and diamagnetic corrections for the ligand moiety were made by using Pascal's constants [7]. A Beckman IR $20 \mathrm{~A}$ spectrophotometer was used for recording the infrared spectra using the $\mathrm{KBr}$ or $\mathrm{CsBr}$-disc technique. Electronic spectra were recorded in solution with the help of a Cary $17 \mathrm{D}$ spectrophotometer whereas, mull spectra of the compounds (in Nujol) were recorded in a Hungarian MOM 201 spectrophotometer, using the technique of Lee et al. [8].

\section{Results and Discussion}

The analytical results revealed that in the first compound (A), the metal:ligand ratio was $1: 3$ whereas, in case of the other two compounds metal:ligand ratio was 1:2. Oxidation state of manganese in each of the above compounds was determined by oxidimetric procedures using both acidified potassium iodide and standard Mohr salt solutions. In all cases it was found to be +3 . This conclusion was supported by the magnetic moment values of the chelates ( $\mu_{\text {eff }}: 3.9-4.0$ B.M.) recorded at $298 \mathrm{~K}$ which are characteristic of high-spin octahedral Mn(III) complexes [9]. Electronic absorption spectra of the complexes exhibited the presence of a broad band in the region $15,990-19,850 \mathrm{~cm}^{-1}$ (corresponding to the transition ${ }^{5} \mathrm{E}_{\mathrm{g}} \rightarrow{ }^{5} \mathrm{~T}_{2 \mathrm{~g}}$, with $\varepsilon$ between $40-60 \mathrm{~cm}^{-1} \mathrm{~mole}^{-1}$ ) indicative of a $\mathrm{Mn}$ (III) species in an octahedral surrounding [10].

IR spectra of the ligands showed that both pyridine 2,6-dicarboxylic acid and pyridine-2,4,6-tricarboxylic acid exhibited the - $\mathrm{COO}$ stretch as a strong band at $1720-1715 \mathrm{~cm}^{-1}$ region and pyridine-2carboxylic acid exhibited a strong absorption band at $1710 \mathrm{~cm}^{-1}$, which is due to the same stretching mode $[11,12]$. In the IR spectrum of the first chelate (A) this $1710 \mathrm{~cm}^{-1}$ band is shifted towards lower wave number $1675 \mathrm{~cm}^{-1}$ and is indicative of bonding through the carboxylate moiety [13]. But, in case of the other two chelates the presence of coordinated carboxylate moiety (strong band at $\left.1675-1665 \mathrm{~cm}^{-1}\right)$ [13] as well as that of uncoordinated carboxyl moiety (band around 1720-1715 $\mathrm{cm}^{-1}$ ) [14] have been confirmed from their IR spectra. All the complexes exhibited the presence of lattice water in the forms of rather broad bands at $3500-3400 \mathrm{~cm}^{-1}$ due to $\mathrm{O}-\mathrm{H}$ stretch and at $1630-1600 \mathrm{~cm}^{-1}$ due to $\mathrm{HOH}$ bend [15].

In the IR spectra of all three complexes the two bands at $600-640 \mathrm{~cm}^{-1}$ (in-plane ring deformation) and at $400-415 \mathrm{~cm}^{-1}$ (out-of-plane ring deformation) were shifted to higher frequency region by $20-35$ wave numbers and this has been considered as evidence of coordination through the heterocyclic nitrogen $[16,17]$. Analytical results, electronic and IR spectral data and room temperature magnetic moment values indicate an octahedral disposition of the donor sites around high-spin $\mathrm{Mn}$ (III) ion. All three complexes are remarkably stable even in nearly boiling aqueous solutions and can be recrystallised several times from water without significant decomposition. The complex (A) is the most stable one of the three. A dilute aqueous solution of this complex, in presence of a little extra ligand, is stable for several weeks. It does not decompose on refluxing for hours with alcohols or acetone.

At the end, a very interesting finding may be emphasised upon. All three complexes (A), (B) and (C) have been obtained from $\mathrm{MnO}_{2}$, a tetravalent manganese compound. Again, the compound (A) 
has also been synthesised from heptavalent manganese by heating $\mathrm{KMnO}_{4}$ with an aqueous solution of picolinic acid. All of them have also been prepared from $\mathrm{Mn}$ (III) compound like $\mathrm{Mn}$ (III) acetate and the compound (A). Hence, these three ligands appear to have a special capacity for stabilising the $\mathrm{Mn}$ (III) state.

Utilisation of these three $\mathrm{Mn}$ (III) chelates as oxidants for effecting oxidation of suitable organic and inorganic substrates are in progress.
[1] M. M. Ray and P. Ray, J. Indian Chem. Soc. 35, 595 (1958).

[2] R. S. Nyholm and G. J. Sutton, J. Chem. Soc. 1958, 564.

[3] R. S. Nyholm and A. Turco, Chem. Ind. 1960, 74.

[4] R. S. Nyholm and A. Turco, J. Chem. Soc. 1962, 1121.

[5] A. B. P. Lever, J. Lewis, and R. S. Nyholm, J. Chem. Soc. 1962, 5262.

[6] M. M. Ray, J. N. Adhya, D. Biswas, and S. N. Poddar, Aust. J. Chem. 19, 881 (1966).

[7] P. W. Selwood, Magneto Chemistry, Interscience Publishers, New York 1956, p. 52.

[8] R. H. Lee, E. Griswold, and J. Kleinberg, Inorg. Chem. 3, 1278 (1964).

[9] B. N. Figgis and J. Lewis, Modern Coordination Chemistry, Interscience Publishers, U.S.A. 1967, p. 407.
[10] A. B. P. Lever, Inorganic Electronic Spectroscopy, Elsevier, Amsterdam 1968, p. 291.

[11] S. Ghosh, T. K. Banerjee, and P. K. Ray, J. Indian Chem. Soc. 55, 610 (1978).

[12] R. L. Dutta and S. Ghosh, J. Indian Chem. Soc. 44, 377 (1967).

[13] K. Nakamoto, Y. Korimoto, and A. E. Martell, J. Am. Chem. Soc. 84, 2081 (1962).

[14] J. A. Kieft and K. Nakamoto, J. Inorg. Nucl. Chem. 29, 2561 (1967).

[15] K. Nakamoto, Infrared Spectra of Inorganic and Coordination Compounds, Interscience Publishers, New York 1970, p. 166.

[16] R. J. H. Clark and C. S. Williams, Inorg. Chem. 4, 350 (1965).

[17] N. S. Gill, R. H. Nuttal, D. E. Scaife, and D. W. A. Sharp, J. Inorg. Nucl. Chem. 18, 79 (1961). 